\title{
A Linear Regression Approach for Determining Explicit Expressions for Option Prices for Equity Option Pricing Models with Dependent Volatility and Return Processes
}

\author{
Raj Jagannathan \\ Department of Management Sciences, Tippie College of Business, The University of lowa, lowa City, \\ IA, USA \\ Email: raj-jagannathan@uiowa.edu
}

Received 28 June 2015; accepted 16 May 2016; published 19 May 2016

Copyright (C) 2016 by author and Scientific Research Publishing Inc.

This work is licensed under the Creative Commons Attribution International License (CC BY).

http://creativecommons.org/licenses/by/4.0/

(c) (i) Open Access

\begin{abstract}
We consider a risk-neutral stock-price model where the volatility and the return processes are assumed to be dependent. The market is complete and arbitrage-free. Using a linear regression approach, explicit functions of risk-neutral density functions of stock return functions are obtained and closed form solutions of the corresponding Black-Scholes-type option pricing results are derived. Implied volatility skewness properties are illustrated.
\end{abstract}

\section{Keywords}

Option Pricing, Black-Scholes Model, Heston's Model, Risk-Neutral Density Functions, Linear Regression Approach, Implied Volatility Functions, Ito Formula

\section{Introduction}

Stochastic volatility (SV) modeling is the subject of several papers in the option price literature. By assuming that the volatility and the return processes of a stock price model are correlated, one can explain better the skewness of the implied volatility curve. Apart from the single-factor CEV model [1], the models proposed are mostly variations of 2-factor affine-jump diffusion models, [2]-[4], with one of the factors being stock volatility. The 2-factor affine model [2] assumes correlated volatility and asset return processes. In [2], however, one has to numerically integrate conditional characteristic functions obtained as solutions of nonlinear $p d f$ to derive the call option prices. The case of the two factors, namely the asset price and volatility being uncorrelated, is considered 
in the paper [5], which obtains Call Option Price Conditional on the variance rate $\bar{V}^{2}$ and derives the unconditional call price by integrating using an approximate probability density function $g(\bar{V})$. The paper [6] considers stochastic forward rate processes which are lognormally distributed conditional on the volatility state variables. See also [7] pp 182-183, for other numerical approximation methods.

Some of the well-known numerical procedures for deriving option pricing that are tree-based binomial or treebased trinomial are available in [8] and [9]. GARCH based heteroscedacity models are discussed in [10]-[13] where empirical versions of SV models in discrete time are approached.

In the next section, the proposed two-factor stock price model that allows the volatility factor and the Brownian motion return processes to be dependent and a linear regression approach that derives explicit expressions for the distribution functions of log return of a stock or stock index are used.

In the subsequent section, we obtain a closed form formula for the call option price that has an algebraic expression that is similar to that of a Black-Scholes model, making it much easier to compute its value.

In the following section, we define an implied volatility function and derive its skewness property.

Finally, we provide concluding remarks and suggestions for future direction.

\section{Heston's Stochastic Volatility Model}

It is known that under a Black-Scholes model formulation the implied volatility function must remain constant for different values of the strike price when the other parameters of the option pricing model are kept constant. However, skewness in implied volatility curves is observed in actual market data for European options. To explain the skewness property of implied volatility functions, [2] considers the following model (1)-(3) with the condition that the (2) asset price and volatility are correlated:

$$
\begin{aligned}
& \frac{\mathrm{d} S(t)}{S(t)}=\mu \mathrm{d} t+\sqrt{v_{t}} \mathrm{~d} z_{1}(t) \\
& \mathrm{d} \sqrt{v_{t}}=-\beta \sqrt{v_{t}}+\delta \mathrm{d} z_{2}(t) \\
& \operatorname{Cov}\left(\mathrm{d} z_{1}(t), \mathrm{d} z_{2}(t)\right)=\rho \mathrm{d} t
\end{aligned}
$$

where $z_{j}, j=1,2$ are Brownian processes.

Note that it can be shown, applying the Ito formula, that the variance rate $v_{t}$ has a square root process model (see [2]).

Computation of option price in the case of the above correlated model as described in using a $p d f$ is fairly complicated. To obtain a closed form solution for the option price one has to invert two conditional characteristic functions to compute the difference between two probability functions as the required solution of the $p d f$.

\section{A Two-Factor Stochastic Volatility Model}

Here, we will explicitly specify the $s d e$ of the asset price and volatility processes. In this paper, we consider a risk-adjusted diffusion process (4) for spot asset price $X(u), 0 \leq u \leq s$, defined with respect to a probability space $\left(\Omega, \Gamma_{u}, P\right)$, with the data-gathering measure $P$

$$
\begin{gathered}
\frac{\mathrm{d} X(u)}{X(u)}=\mu \mathrm{d} u+v \mathrm{~d} B(u) ; \\
\mathrm{d} \ln X(u)=\left(\mu-v^{2} / 2\right) \mathrm{d} u+v \mathrm{~d} B(u)
\end{gathered}
$$

In (4), $v \in \mathrm{R}$ is called the instantaneous diffusion rate and $\mu$ is called the instantaneous drift rate of the diffusion process.

In (5), we have a log normal model for the asset price $X(u), 0 \leq u \leq s$.

At this point, we introduce a second factor $H(u)$, which is a mean-reverting process, in Equation (7), and corresponds to the volatility $\sqrt{v_{t}}$ in Equation (1) of Heston's model. 


$$
\begin{gathered}
\frac{\mathrm{d} X(u)}{X(u)}=\left(\mu+\frac{H^{2}(u)}{2}\right) \mathrm{d} u+(H(u)+v) \mathrm{d} B(u) \\
\mathrm{d} H(u)=\alpha(H(u)-\theta)+\eta \mathrm{d} Y(u), \theta, \eta \geq 0 \text { and } \alpha>0 .
\end{gathered}
$$

(6) can be transformed to

$$
d \ln X(u)=\left(\mu-\frac{v^{2}}{2}\right) \mathrm{d} u+(H(u)+v) \mathrm{d} B(u)
$$

\section{Formulation of a Risk-Neutral Model}

The dynamic processes (8)-(9) below are defined with respect to the martingale probability measure $Q$, where $B_{1}(u)$ and $Y_{1}(u)$ are Brownian motions under $Q$, where we assume the corresponding Novikov's condition is satisfied.

\section{Two Factor Risk-Neutral Model}

$$
\frac{\mathrm{d} X(u)}{X(u)}=\left(r+\frac{H^{2}(u)}{2}\right) \mathrm{d} u+(H(u)+v) \mathrm{d} B_{1}(u)
$$

\section{Remark 1:}

An equivalent Two-factor Black-Derman-Toy model [14] can be formulated.

The sde (6) and (8) can be transformed using Ito formula to (6a) and (9), a two-factor Black-Derman-Toy (1990)-type model [14] obtained by introducing a second factor $H(u)$ in Equation (5).

As mentioned previously, in (4), $v \in \mathrm{R}$ is the instantaneous diffusion rate and $\mu$ is called the instantaneous drift rate of the diffusion process.

As stated previously, in Equation (7), we define the volatility $H(u) \in \mathrm{R}$ as a mean reverting Gaussian process with $\theta$ as its long-term mean ${ }^{1}$.

We assume $H(u)$ to be correlated with $B_{1}(u)$ as in the Equation (8) and that $Y_{1}(u)$ is a standard Brownian motion process.

Then it follows (see [2]).that the distribution of $H(u)$ is:

$$
H(u) \sim N\left(q(u), \frac{\eta}{\sqrt{2 \alpha}}\left(1-\mathrm{e}^{-2 \alpha u}\right)^{1 / 2}\right), 0 \leq u \leq s .
$$

Alternatively, $H(u)$ may be expressed as

$$
H(u)=q(u)+\int_{0}^{u} \psi(t)\left[\rho \mathrm{d} B_{1}(t)+\delta \mathrm{d} C_{1}(t)\right] \mathrm{d} B_{1}(u)
$$

where

$$
\begin{aligned}
& \psi(t)=\eta \mathrm{e}^{-\alpha t}, \eta, \alpha \geq 0,0 \leq t \leq u, \\
& q(u) \triangleq \kappa \mathrm{e}^{-\alpha u}+\theta\left(1-\mathrm{e}^{-\alpha u}\right) .
\end{aligned}
$$

Assumption 1: The Brownian motion processes $Y_{1}(u)$ and $B_{1}(u)$ are related as follows:

$$
Y_{1}(u)=\rho B_{1}(u)+\delta C_{1}(u),
$$

where $\delta=\sqrt{1-\rho^{2}}$.

Also, the Brownian motion processes $B_{1}(u)$ and $C_{1}(u)$ under $Q$ are independent.

${ }^{1}$ This process is known as " $O-U$ " process, the Ornstein-Uhlenbeck process. 
See [15] for a similar assumption. See also [3] and [4].

From (6) and (10), it is clear that

$$
\begin{gathered}
\mathrm{d} \ln X(u)=\left(r-\frac{v^{2}}{2}\right) \mathrm{d} u+\left(v+q(u)+\int_{0}^{u} \psi(t)\left[\rho \mathrm{d} B_{1}(t)+\delta \mathrm{d} C_{1}(t)\right]\right) \mathrm{d} B_{1}(u) ; \\
\delta \triangleq \sqrt{1-\rho^{2}} \\
\psi(u)=\eta \mathrm{e}^{-\alpha u}, \eta, \alpha \geq 0 .
\end{gathered}
$$

Equation (11) follows because from [16] we know that the Gaussian random variable $H(u)$ may be expressed as

$$
H(u)=q(u)+\int_{0}^{u} \psi(t)\left[\rho \mathrm{d} B_{1}(t)+\delta \mathrm{d} C_{1}(t)\right], 0 \leq u \leq s,
$$

where

$$
\begin{aligned}
& \delta \triangleq \sqrt{1-\rho^{2}}, \\
& \psi(t)=\eta \mathrm{e}^{-\alpha t}, \eta, \alpha \geq 0, \\
& H(0)=\kappa \\
& E H(u) \triangleq q(u) \triangleq \kappa \mathrm{e}^{-\alpha u}+\theta\left(1-\mathrm{e}^{-\alpha u}\right)=\theta+q_{0}(u), \\
& \text { where } q_{0}(u) \triangleq(\kappa-\theta) \mathrm{e}^{-\alpha u} \\
& \operatorname{VarH}(u) \triangleq \sigma_{H}^{2}(u)=\int_{0}^{u} \psi^{2}(t) \mathrm{d} t=\eta^{2} \frac{\left(1-\mathrm{e}^{-2 \alpha u}\right)}{2 \alpha}
\end{aligned}
$$

Note that $B_{1}(s)$ has a normal distribution with mean 0 and variance $s$, so $B_{1}(s)$ can be written as $B_{1}(s)=Z(s) \sqrt{s}$, where $Z(s)$ is a standard normal variable. Then $\ln X(s)$ can be written as a quadratic function of $Z(s)=\frac{B_{1}(s)}{\sqrt{s}}, s>0$. plus a residual term $\varepsilon(s)$. (See Proposition 1 below).

For $\mathrm{d} \xi=\mathrm{d} C(t), 0 \leq t \leq u, 0 \leq u \leq s$, we define a volatility process

$$
\begin{gathered}
V(\xi)=\left\{V_{u}=\int_{0}^{u} \psi(t) \mathrm{d} C(t), 0 \leq u \leq s\right\} . \\
\mathrm{d}[\ln X(u)]=\left(r-\frac{v^{2}}{2}\right) \mathrm{d} u+\left[v+\theta+q_{0}(u)+\int_{0}^{u} \psi(t)\left(\rho \mathrm{d} B_{1}(t)+\delta \mathrm{d} C_{1}(t)\right)\right] \mathrm{d} B_{1}(u), \\
\int_{0}^{s} \mathrm{~d}[\ln X(u)]=\int_{0}^{s}\left(r-\frac{v^{2}}{2}\right) \mathrm{d} u+\int_{0}^{s}\left(v+\theta+q_{0}(u)\right) \mathrm{d} B_{1}(u)+\int_{0}^{s} \int_{0}^{u} \psi(t) \rho \mathrm{d} B_{1}(t) \mathrm{d} B_{1}(u) \\
+\int_{0}^{s} \int_{0}^{u} \psi(t) \delta \mathrm{d} C_{1}(t) \mathrm{d} B_{1}(u), 0 \leq t \leq u \leq s
\end{gathered}
$$

Define $\sigma(V(s)) \triangleq\left(\frac{1}{s} \int_{0}^{s}\left[\frac{\eta^{2}\left(1-\mathrm{e}^{-2 \alpha u}\right)}{2 \alpha}\right] \mathrm{d} u\right)^{1 / 2} \triangleq \bar{V}$ as the average standard deviation in the case of uncorrelated Brownian motion process $\left\{C_{1}(u), 0 \leq u \leq s\right\} \triangleq \xi$. (see [7], p. 182). 
Then the average variance is:

$$
\sigma^{2}(V(s))=\int_{0}^{s}\left[\frac{\eta^{2}\left(1-\mathrm{e}^{-2 \alpha u}\right)}{2 \alpha}\right] \mathrm{d} u=\frac{1}{s} \int_{0}^{s}\left(\frac{\eta^{2}\left(1-\mathrm{e}^{-2 \alpha u}\right)}{2 \alpha}\right)^{2} \mathrm{~d} u=\frac{1}{s} \frac{\eta^{2}\left(2 \alpha s-\left(1-\mathrm{e}^{-2 \alpha s}\right)\right)}{4 \alpha^{2}} \triangleq V^{2} .
$$

\section{Proposition 1:}

$$
\begin{aligned}
\int_{0}^{s} \mathrm{~d} \ln X(u)=\int_{0}^{s}\left(r-\frac{v^{2}}{2}\right) \mathrm{d} u+\int_{0}^{s}\left[v+\theta+q_{0}(u)\right] \mathrm{d} B_{1}(u)+\int_{0}^{u}\left[\psi(t)\left(\rho d B_{1}(t)+\delta C_{1}(t)\right)\right] \mathrm{d} B_{1}(u) ; \\
\ln X_{s}=\ln x_{0}+r s-\frac{1}{2} v^{2} s+\left[(v+\theta) B_{1}(s)+\left[\gamma_{1}(s) B_{1}(s)+e_{1}(s)\right]\right. \\
\left.+\left[\gamma_{2}(s) \rho\left(\frac{B_{1}^{2}(s)}{2}-\frac{s}{2}\right)+e_{2}(s)\right]+\delta \bar{V} B_{1}(s)\right] \\
\triangleq \\
Z^{2}(s) \frac{m(s)}{2}+Z(s) n(s)+p(s)+\varepsilon(s) \triangleq U(s, \xi)+\varepsilon(s),
\end{aligned}
$$

and where

$$
U(s, \xi)=Z^{2}(s) \frac{m(s)}{2}+Z(s) n(s)+p(s),
$$

$m(s)=\rho \gamma_{2}(s) s$

$$
\begin{aligned}
& n(s)=\left(\gamma_{1}(s)+v+\theta+\delta \bar{V}\right) \sqrt{s}, \\
& p(s)=\ln x_{0}+\left(r-\frac{1}{2} v^{2}\right) s-\rho \gamma_{2}(s) \frac{s}{2}
\end{aligned}
$$

$\varepsilon(s)=e_{1}(s)+e_{2}(s)$, where

$$
\begin{aligned}
& \gamma_{1}(s)=\frac{1}{s} \int_{0}^{s} q_{0}(u) \mathrm{d} u=\frac{(\kappa-\theta)\left(1-\mathrm{e}^{-\alpha s}\right)}{\alpha s} \\
& \gamma_{2}(s)=\frac{2 \eta}{s^{2}} \int_{0}^{s} \int_{0}^{u} \psi(t) \mathrm{d} t \mathrm{~d} u=\frac{2 \eta}{s^{2}} \int_{0}^{s} \frac{\left(1-\mathrm{e}^{-\alpha u}\right)}{\alpha} \mathrm{d} u=\frac{2 \eta}{s^{2}} \frac{\left(\alpha s-\left(1-\mathrm{e}^{-\alpha s}\right)\right)}{\alpha^{2}} .
\end{aligned}
$$

$\varepsilon(s) \doteq N\left(0, \sigma_{\varepsilon}\right)$, approximately, where

$$
\begin{gathered}
\sigma_{\varepsilon}=\sqrt{\left(\operatorname{var}\left(e_{1}(s)\right)+\operatorname{var}\left(e_{2}(s)\right)\right)}, \text { because } \\
\operatorname{Cov}\left(e_{1}(s), e_{2}(s)\right)=0 \\
\operatorname{Var}\left(e_{1}(s)\right)=(\kappa-\theta)^{2}\left[\left(\frac{1-\mathrm{e}^{-2 \alpha s}}{2 \alpha}\right)-\left(\frac{1-\mathrm{e}^{-\alpha}}{\alpha s}\right)^{2}\right] \\
\operatorname{Var}\left(e_{2}(s)\right)=\left\{\frac{\eta^{2} s}{2 \alpha}-\frac{\eta^{2}}{4 \alpha^{2}}\left(1-\mathrm{e}^{-2 \alpha s}\right)\right\}-\left\{\frac{\eta s}{\alpha}-\frac{\eta}{\alpha^{2}}\left(1-\mathrm{e}^{-\alpha s}\right)\right\}^{2} \\
\operatorname{Cov}\left(e_{1}(s), e_{2}(s)\right)=0
\end{gathered}
$$

Proof: See Appendix A

Assumption 2: 
(a) $\varepsilon(s)$ and $U(s, \xi)$ are independent random variables.

(b) $\varepsilon(s) \doteq N\left(0, \sigma_{\varepsilon}\right)$ approximately where

$$
\sigma_{\varepsilon}=\sqrt{\left(\operatorname{var}\left(e_{1}(s)\right)+\operatorname{var}\left(e_{2}(s)\right)\right)} \text {, because } \operatorname{Cov}\left(e_{1}(s), e_{2}(s)\right)=0
$$

\section{Remark 2:}

Some of the limitations of the model can be described as follows:

a) Since we can verify that $C \operatorname{ov}(\varepsilon(s), U(\xi, s))=0$, we have only the necessary condition for independence between $\varepsilon(s)$ and $U(s, \xi)$ is satisfied.

b) We have assumed that the error terms $e_{1}(s)$ and $e_{2}(s)$ of the linear regressions are normally distributed and that $\varepsilon(s)=e_{1}(s)+e_{2}(s)$ is also normally distributed.

\section{Proposition 2:}

$$
E_{0}^{Q}\left(\mathrm{e}^{-r X(s)}\right)=x_{0}
$$

where the expectation is obtained using the risk neutral distribution of $X(s)$, as defined in (6).

\section{Remark 3:}

Proposition 2 restates the result that the risk neutral property of $X(t), t \geq 0$. holds; the normalized process $\mathrm{e}^{-r s} X(s), s \geq 0$, is a martingale with respect to $Q$ and the market $\{X(u), u \geq 0\}$ is arbitrage free.

\section{Derivative Securities}

We can evaluate any security that is a derivative of $X(s)$ using the risk neutral probability distribution of $X(s)$. In particular, consider a non-dividend paying European call option with strike price $K$ and maturity dates ${ }^{2}$.

Then the price $C\left(x_{0}, \rho, K, r, \eta^{2}, s\right)$ at time 0 of the call option is the present value of the expected terminal value, $E_{0}^{Q}\left(\mathrm{e}^{-r s}(X(s)-K)^{+}\right)$, where the expectation is obtained using the risk neutral distribution of $X(s)$.Similarly the put option is defined as $P\left(x_{0}, \rho, K, r, \eta^{2}, s\right)=E_{0}^{Q}\left(\mathrm{e}^{-r s}(X(s)-K)^{-}\right)$. Then, using Put Callparity formula and the Equation (18) we have

$$
\begin{gathered}
C\left(x_{0}, \rho, K, r, \eta^{2}, s\right)-P\left(x_{0}, \rho, K, r, \eta^{2}, s\right)=E_{0}^{Q}\left(\mathrm{e}^{-r s}\left(X_{s}-K\right)\right)=x_{0}-\mathrm{e}^{-r s} K \\
\therefore P\left(x_{0}, \rho, K, r, \eta^{2}, s\right)=C\left(x_{0}, \rho, K, r, \eta^{2}, s\right)-\left(x_{0}-\mathrm{e}^{-r s} K\right)
\end{gathered}
$$

In the next sections, we will derive a simple Black-Sholes type expression for the call option price $C\left(x_{0}, \rho, K, r, \eta^{2}, s\right)$ and derive its properties.

\section{Implied Risk-neutral distribution of $\ln X(s)$}

For easier reference we present below the explicit expressions for the vector

$$
\begin{aligned}
& \quad(m(s), n(s), p(s), \varepsilon(s)) \\
& m(s)=\rho \gamma_{2}(s) s ; \\
& n(s)=\left(\gamma_{1}(s)+v+\theta+\delta \bar{V}\right) \sqrt{s} \\
& p(s)=\ln x_{0}+\left(r-\frac{1}{2} v^{2}\right) s-\rho \gamma_{2}(s) \frac{s}{2}=\ln x_{0}+\left(r-\frac{1}{2} v^{2}\right) s-m(s) / 2 ; \\
& \varepsilon(s)=e_{1}(s)+e_{2}(s) ;
\end{aligned}
$$

where the conditional risk-neutral distribution function of $(\ln X(s) \mid \varepsilon(s)=h)$ is derived below.

\footnotetext{
${ }^{2}$ This condition can be relaxed by replacing $r$ by $r-d$, where $d$ is the dividend payout rate and $r$ is the annual risk-free interest rate.
} 
Next we determine an explicit expression for the conditional distribution function

$$
F_{\ln X(s) \xi}(\omega, x)=P(\ln X(s) \leq \omega \mid \varepsilon(s)=h), \omega \in \mathrm{R}
$$

So given $\varepsilon(s)=h$

$$
\begin{aligned}
& \ln X(s)-\omega=Z^{2}(s) \frac{m(s)}{2}+Z(s) n(s, \xi)+p(s)+h, \\
& m(s) \neq 0 .
\end{aligned}
$$

Then the roots of the equation

$$
\begin{aligned}
& \ln X(s)-\omega=Z^{2}(s) \frac{m(s)}{2}+Z(s) n(s, \xi)+p(s)+h=0, \\
& m(s) \neq 0 .
\end{aligned}
$$

are

$$
z=z_{1}(h), z_{2}(h)=\frac{-n(s) \pm \sqrt{n^{2}(s)-2 m(s)(p(s)+h)}}{m(s)}
$$

\section{Assumption 3:}

$$
n^{2}(s, \xi)-2 m(s)(p(s)+h) \geq 0
$$

Assumption (3) ensures that the roots are real and are well defined.

Let $F_{\ln X(s) \xi}(\omega, h, \xi)=P(\ln X(s) \leq \omega \mid \varepsilon(s)=h, \bar{V}(s)=\xi)$

Then

where

$$
\ln X(s)-\omega=\frac{m(s)}{2}\left(Z-z_{1}\right)\left(Z-z_{2}\right)
$$

$$
\begin{gathered}
z=z_{1}(h-\omega), z_{2}(h-\omega)=\frac{-n(s) \pm \sqrt{n^{2}(s, \xi)-2 m(s)(p(s)+h-\omega)}}{m(s)} \\
n^{2}(s, \xi)-2 m(s)(p(s)+h-\omega) \geq 0 \Rightarrow \omega \geq \omega^{*}(\rho, h, \xi)=h+p(s)-\frac{n^{2}(s, \xi)}{2 m(s)} \\
p(s)=\ln x_{0}+\left(r-\frac{1}{2} v^{2}\right) s-\rho \gamma_{2}(s) \frac{s}{2}
\end{gathered}
$$

Define

$$
p^{*}(s, h) \triangleq p(s)+h-r s-\ln x_{0}+\ln K
$$

and also suppose Assumption (2) holds. Note that the functions $m(s)$ and $n(s)$ are independent of $h$.

Conditional Risk-neutral distribution of $(\ln X(s) \mid \varepsilon(s)=h, \bar{V}(s)=\xi), m(s)>0$

\section{Remark 4:}

If $m(s)>0$, then $(\ln X(s) \mid \varepsilon(s)=h, \bar{V}(s)=\xi)$ is a convex function of $Z(s)$ and a minimum of $\ln (X(s))$ as a function of $Z(s)$ exists.

Similarly if $m(s)<0$, then $\ln X(s) \mid(\varepsilon(s)=h, \bar{V}(s)=\xi)$ is a concave function of $Z(s)$ and a maximum of $\ln (X(s))$ as a function of $Z(s)$ exists.

\section{Proposition 3:}


Suppose $\rho>0$, which implies that $m(s)>0$.

If Assumption (3) holds then the conditional risk-neutral distribution of $\{\ln X(s) \mid \varepsilon(s)=h, \bar{V}(s)=\xi\}$ is:

$$
\begin{aligned}
& 1-F_{\ln X(s)}(\omega, h)=P(\ln X(s) \geq \omega \mid \varepsilon(s)=h)=\left\{\begin{array}{l}
1-\Phi\left(z_{1}(h)\right)+\Phi\left(z_{2}(h)\right), \omega \geq \omega^{*}(\rho, h, \xi) \\
1, \omega \leq \omega^{*}(\rho, h, \xi)
\end{array}\right) \\
& \therefore F_{\ln X(s)}(\omega, h)=P(\ln X(s) \leq \omega \mid \varepsilon(s)=h)=\left\{\begin{array}{l}
\Phi\left(z_{1}(h)\right)-\Phi\left(z_{2}(h)\right), \omega \geq \omega^{*}(\rho, h, \xi) \\
0, \omega \leq \omega^{*}(\rho, h, \xi)
\end{array}\right. \\
& \text { where } z=z_{1}(h, \xi), z_{2}(h, \xi)=\frac{-n(s) \pm \sqrt{n^{2}(s, \xi)-2 m(s)(p(s)+h-\omega)}}{m(s)} \\
& \omega^{*}(\rho, h)=h+p(s)-\frac{n^{2}(s)}{2 m(s)}
\end{aligned}
$$

If $n^{2}(s, \xi)-2 m(s)\left(p(s)-h-\omega^{*}\right)=0$, then the roots of the equation defined in (18) are equal so that $z_{1}=z_{2}=z^{*}(\xi)=-\frac{n(s, \xi)}{m(s)}$, then there exists a value $\omega^{*}(\rho, h, \xi)$ such that $P\left(\ln X(s) \geq \omega^{*}(\rho, h) \mid \varepsilon(s)=h, \tilde{V}^{2}(s)=\xi\right)=1$.

In other words, $\omega^{*}(\rho, h, \xi)$ is the lowest value the conditional random variable $\{\ln X(s) \mid \varepsilon(s)=h\}$ can assume in this case.

Next we consider the case of $m(s)<0$.

Conditional Risk-neutral Distribution function of $(\ln X(s) \mid \varepsilon(s)=h, \bar{V}(s)=\xi), m(s)<0$.

Suppose $\rho<0$, which implies $m(s)<0$.

If Assumption (3) holds then the conditional risk-neutral distribution of $\ln X(s) \mid\left(\varepsilon(s)=h, \bar{V}^{2}(s)=\xi\right)$ is derived as follows:

$$
\begin{aligned}
& 1-F_{\ln X(s) \xi}(\omega, h)=P(\ln X(s) \geq \omega \mid \varepsilon(s)=h, \bar{V}(s)=\xi)=\left\{\begin{array}{l}
\Phi\left(z_{2}(h)\right)-\Phi\left(z_{1}(h)\right), \omega \leq \omega^{*}(\rho, h, \xi) \\
0, \omega \geq \omega^{*}(\rho, h, \xi)
\end{array}\right. \\
& \therefore F_{\ln X(s) \xi}(\omega, h)=\left\{\begin{array}{l}
1-\Phi\left(z_{2}(h)\right)+\Phi\left(z_{1}(h)\right), \omega \leq \omega^{*}(\rho, h, \xi) \\
1, \omega \geq \omega^{*}(\rho, h)
\end{array}\right. \\
& \text { where } z=z_{1}(h, \xi), z_{2}(h, \xi)=\frac{-n(s, \xi) \pm \sqrt{n^{2}(s, \xi)-2 m(s)(p(s)+h-\omega)}}{m(s)}
\end{aligned}
$$

\section{Example 1:}

Let $(r, v, \alpha, \eta, \theta, \rho, \kappa)=(0.06,0.08,0.20,0.5,-0.8,0.1)$. Then in Figure 1 depicts the conditional risk-neutral distribution of

$$
(\ln X(s) \mid \varepsilon(s)=h, \bar{V}(s)=\xi), m(s)<0 .
$$

In the next section we consider the evaluation of price of a security that is derivative of stock price $X(s)$. We need the following Assumption (4) to ensure that the call option price is well defined.

\section{Example 2:}

Let $(r, v, \alpha, \eta, \theta, \rho, \kappa)=(0.06,0.08,0.20,0.5,0.3,0.8,0.1)$.

Figure 2 shows the conditional risk-neutral distribution of $(\ln X(s) \mid \varepsilon(s)=h, \bar{V}(s)=\xi), m(s)>0$. is depicted. 


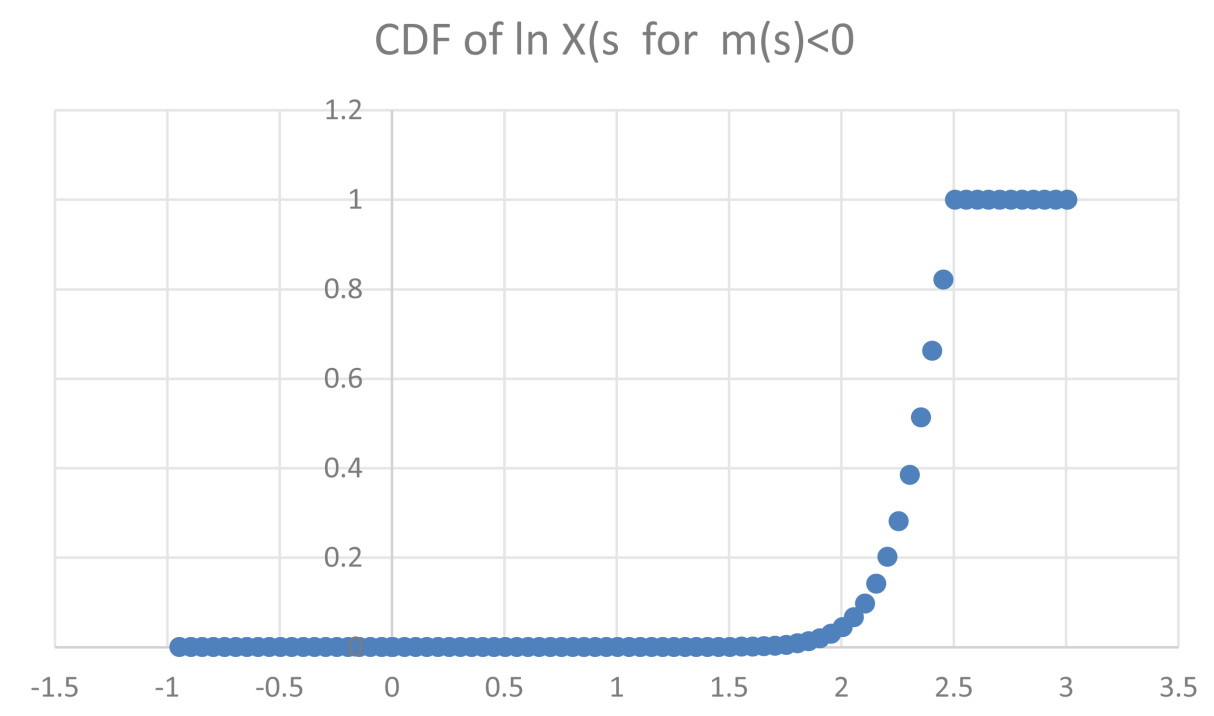

Figure $1.1 \leftarrow \omega \rightarrow \omega^{*}(\rho, h)=1.4084, h=0$.

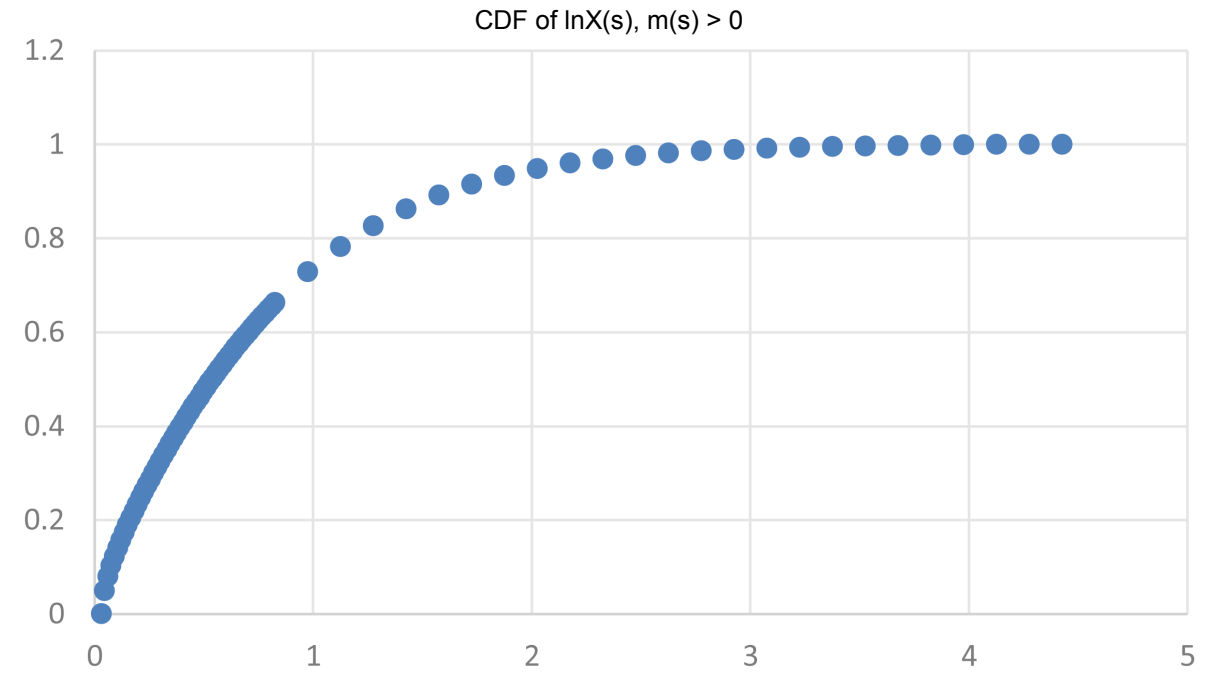

Figure 2. Conditional risk-neutral distribution $m(s)>0$.

\section{Assumption 4:}

We will utilize the Assumption (4) later for deriving the price of any derivative security.

\section{Conditional Call Option Price}

\section{Assumption 4}

$$
m(s)<1 \text {. }
$$

We will utilize the Assumption (4) later for deriving the price of any derivative security.

\section{Conditional Call Option Price}

Next we obtain an explicit closed form expression for the conditional call option price that is similar to the corresponding B-S expression and hence is easier to compute.

\section{Proposition 4:}

Given $\rho>0$ and

$$
\{0<m(s)<1\}
$$




$$
\begin{aligned}
& C^{*}\left(x_{0}, K, r, \eta, h, \rho>0\right) \\
& =E_{0}^{Q}\left(\mathrm{e}^{-r s}(X(s)-K)^{+} \mid \varepsilon(s)=h\right) \\
& \left.=\left\{\begin{array}{l}
2 \cdot \frac{x_{0}}{\sqrt{1-m(s)}}\left\{1-\Phi\left(\frac{x_{9}}{\sqrt{1-m(s)}} \exp \left(p^{*}(s, h)+\frac{n^{2}(s)}{2(1-m(s))}\right)-K \mathrm{e}^{-r s}, \text { for } \ln K \leq \omega^{*}(\rho, h) ;\right.\right. \\
\times \exp \left(p^{*}(s, h)+\frac{n^{2}(s)}{2(1-m(s))}\right)-K(s)
\end{array}\right)+\Phi\left(\frac{z_{2}(h)(1-m(s))-n(s)}{(1-m(s))^{-r s}}\right)\right\}
\end{aligned}
$$

where $p^{*}(s, h) \triangleq p(s, h)-r s-\ln x_{0}+\ln K ; \quad$ and

$$
p(s, h) \triangleq\{p(s) \mid \varepsilon(s)=h\} \text {. }
$$

$z=z_{1}(h-\omega), z_{2}(h-\omega)=\frac{-n(s) \pm \sqrt{n^{2}(s, \xi)-2 m(s)(p(s)+h-\omega)}}{m(s)}$

$n^{2}(s, \xi)-2 m(s)(p(s)+h-\omega) \geq 0 \Rightarrow \omega \geq \omega^{*}(\rho, h, \xi)=h+p(s)-\frac{n^{2}(s, \xi)}{2 m(s)}, \Phi(z)=P(Z \leq z)$ is the $c d f$ of the standard normal variable $Z$.

\section{Remark 5:}

To simplify the presentation of the results, we have suppressed usually the dependence of $n(s, \xi)$ on $\xi$.

\section{Proof:}

$$
\begin{aligned}
& C^{*}\left(x_{0}, K, r, \eta, h, \rho>0\right) \\
& =E_{0}^{Q}\left(\mathrm{e}^{-r s}(X(s)-K)^{+} \mid \varepsilon(s)=h\right) \\
& =\left\{\begin{array}{l}
2 \cdot \frac{x_{9}}{\sqrt{1-m(s)}} \exp \left(p^{*}(s, h)+\frac{n^{2}(s)}{2(1-m(s))}\right)-K \mathrm{e}^{-r s}, \text { for } \ln K \leq \omega^{*}(\rho, h), \\
\left.\sqrt{1-m(s)}\left(\frac{z_{1}(h)(1-m(s))-n(s)}{(1-m(s))^{1 / 2}}\right)+\Phi\left(\frac{z_{2}(h)(1-m(s))-n(s)}{(1-m(s))^{1 / 2}}\right)\right\}
\end{array}\right. \\
& \times \exp \left(p^{*}(s, h)+\frac{n^{2}(s)}{2(1-m(s))}\right)-K \mathrm{e}^{-r s}\left\{1-\Phi\left(z_{1}(h)\right)+\Phi\left(z_{2}(h)\right)\right\}, \text { for } \ln K>\omega^{*}(\rho, h) ;
\end{aligned}
$$

We prove Proposition 4 below using the risk-neutral distribution results (Proposition 3) of $\ln X(s)$ for $\rho>0$. Again using the risk-neutral distribution results of $\ln X(s)$ (Proposition3) for $\rho<0$, the Proposition 5 issimilarly proved.

\section{Case 1:}

Here, we make use of risk-neutral distribution of $\ln X(s)$ results for $\rho>0$.

$$
z=z_{1}, z_{2}=\frac{-n(s) \pm \sqrt{n^{2}(s)-2 m(s) p(s)}}{m(s)}
$$

where $m(s) \neq 0, m(s), n(s)$ and $p(s, h)$ are as defined in (19) 


$$
\begin{aligned}
& C^{*}\left(x_{0}, K, r, \sigma, h, \xi\right)=E_{0}^{Q}\left(\mathrm{e}^{-r s}(X(s)-K)^{+} \mid \varepsilon(s)=h, \xi\right) \\
= & E_{0}^{Q}\left(\mathrm{e}^{-r s}\left\{\int_{\ln K}^{\infty} \mathrm{e}^{\ln X(s)} \mathrm{d} F_{\ln (X(s))}-K \int_{\ln K}^{\infty} \mathrm{d} F_{\ln (X(s))}\right\} \mid \varepsilon(s)=h, \xi\right) \\
= & x_{0}\left\{\frac{1}{\sqrt{2 \pi}} \int_{z_{1}}^{\infty} \mathrm{e}^{\frac{(1-m(s))}{2} z^{2}+n(s) z+p^{*}(s, h)} \mathrm{d} z+\frac{1}{\sqrt{2 \pi}} \int_{-\infty}^{z_{2}} \mathrm{e}^{-\left(1-m(s) z^{2}+2 n(s) z+2 p^{*}(s \cdot h)\right.} \mathrm{d} z\right\} \\
& -K \mathrm{e}^{-r s}\left\{\frac{1}{\sqrt{2 \pi}} \int_{z_{1}}^{\infty} \mathrm{e}^{-\frac{z^{2}}{2}} \mathrm{~d} z+\frac{1}{\sqrt{2 \pi}} \int_{-\infty}^{z_{2}} \mathrm{e}^{-\frac{z^{2}}{2}} \mathrm{~d} z\right\} \\
= & x_{0} \frac{1}{\sqrt{1-m(s)}}\left\{1-\Phi\left(\frac{z_{1}(h)(1-m(s))-n(s)}{(1-m(s))^{1 / 2}}\right)+\Phi\left(\frac{z_{2}(h)(1-m(s))-n(s)}{(1-m(s))^{1 / 2}}\right)\right\} \\
& \times \exp \left(p^{*}(s, h)+\frac{n^{2}(s)}{2(1-m(s))}\right)-K \mathrm{e}^{-r s}\left\{1-\Phi\left(z_{1}(h)\right)+\Phi\left(z_{2}(h)\right)\right\}, \text { if } \ln K \geq \omega^{*}(\rho, h) ;
\end{aligned}
$$

\section{Case 2:}

$$
\ln K<\omega^{*}(\rho, h)
$$

Since $P\left(\ln X(s)>\omega^{*}(\rho)\right)=1$, it follows that if $\ln K \leq \omega^{*}(\rho, h)$ then

$$
\begin{array}{r}
P(\ln X(s) \geq \ln K)=1 . \\
C^{*}\left(x_{0}, K, \rho, \sigma^{2}, s, h\right)=E_{0}\left(\mathrm{e}^{-r s}(X(s)-K)^{+} \mid \varepsilon(s)=h\right)
\end{array}
$$

Then, $=E_{0}\left(\mathrm{e}^{-r s}\left\{\int_{-\infty}^{\infty} \mathrm{e}^{\ln X(s)} \mathrm{d} F_{\ln (X(s))}-K \int_{-\infty}^{\infty} \mathrm{d} F_{\ln (X(s))} \mid \varepsilon(s)=h\right\}\right)$ which follows as in Case 1.

$$
=x_{0} \frac{1}{\sqrt{1-m(s)}} \exp \left(p^{*}(s, h)+\frac{n^{2}(s)}{2(1-m(s))}\right)-K \mathrm{e}^{-r s},
$$

This completes the proof.

\section{Proposition 5:}

Suppose $\rho<0$ which $\Rightarrow m(s)<0$ and which easily satisfies the Assumption (4): $m(s)<1$. Then,

$$
\begin{aligned}
& C^{*}\left(x_{0}, K, r, \eta, h, \rho<0\right)=E_{0}^{Q}\left(\mathrm{e}^{-r s}(X(s)-K)^{+} \mid \varepsilon(s)=h\right) \\
& \equiv 0, \text { for } \ln K>\omega^{*}(\rho, h), \\
& =x_{0} \frac{1}{\sqrt{1-m(s)}}\left\{\Phi\left(\frac{z_{2}(h)(1-m(s))-n(s)}{(1-m(s))^{1 / 2}}\right)-\Phi\left(\frac{z_{1}(h)(1-m(s))-n(s)}{(1-m(s))^{1 / 2}}\right)\right\} \\
& \times \exp \left(p^{*}(s, h)+\frac{n^{2}(s)}{2(1-m(s))}\right)-K \mathrm{e}^{-r s}\left\{\Phi\left(z_{2}(h)\right)-\Phi\left(z_{1}(h)\right)\right\}, \text { for } \ln K \leq \omega^{*}(\rho, h) ;
\end{aligned}
$$

Case 1: $\ln K<\omega^{*}(\rho, h)$

Then given that $X(0)=x_{0}$ and $0<m(s)<1$ we have: 


$$
P\left(\ln X(s) \leq \omega^{*}(\rho, h)\right)=1 \Rightarrow P(\ln X(s)<\ln K)=0 .
$$

So in this case

$$
C^{*}\left(x_{0}, K, r, \eta, h, \rho<0\right) \equiv 0,
$$

Case 2: $\ln K>\omega^{*}(\rho, h)$

$$
\begin{aligned}
& \left.C^{*}\left(x_{0}, K, r, \eta, h, \rho>0\right)=E_{0}^{Q}\left(\mathrm{e}^{-r s}(X(s)-K)\right)^{+} \mid \varepsilon(s)=h\right) \\
& =\left\{\begin{array}{l}
x_{0} \frac{1}{\sqrt{1-m(s)}}\left\{\Phi\left(\frac{z_{1}(h)(1-m(s))-n(s)}{(1-m(s))^{1 / 2}}\right)-\Phi\left(\frac{z_{2}(h)(1-m(s))-n(s)}{(1-m(s))^{1 / 2}}\right)\right\} \\
\times \exp \left(p^{*}(s, h)+\frac{n^{2}(s)}{2(1-m(s))}\right)-K \mathrm{e}^{-r s}\left\{\Phi\left(z_{1}(h)\right)-\Phi\left(z_{2}(h)\right)\right\}, \text { for } \ln K>\omega^{*}(\rho, h) ; \\
x_{0} \frac{1}{\sqrt{1-m(s)}} \exp \left(p^{*}(s, h)+\frac{n^{2}(s)}{2(1-m(s))}\right)-K \mathrm{e}^{-r s}, \text { for } \ln K \leq \omega^{*}(\rho, h),
\end{array}\right.
\end{aligned}
$$

\section{Remark 6:}

We define

(i) Hedge ratio $=\frac{\partial C^{*}(.)}{\partial x_{0}}=\Delta($.

Then, given that $X(0)=x_{0}$ and $m(s)<0$ we have:

$$
\begin{aligned}
& \Delta(., K)=\frac{\partial}{\partial x_{0}} C^{*}\left(x_{0}, K, r, \eta, h, \rho<0\right) \\
& =\left\{\begin{array}{l}
\frac{1}{\sqrt{1-m(s)}}\left\{\Phi\left(\frac{z_{2}(h)(1-m(s))-n(s)}{(1-m(s))^{1 / 2}}\right)-\Phi\left(\frac{z_{1}(h)(1-m(s))-n(s)}{(1-m(s))^{1 / 2}}\right)\right\} \\
\times \exp \left(p^{*}(s, h)+\frac{n^{2}(s)}{2(1-m(s))}\right), \text { for } \ln K<\omega^{*}(\rho, h) ; \\
0, \text { for } \ln K \geq \omega^{*}(\rho, h),
\end{array}\right.
\end{aligned}
$$

Figure 3 shows the unconditional hedge ratio as derived using (28).

\section{Unconditional Hedge Ratio}

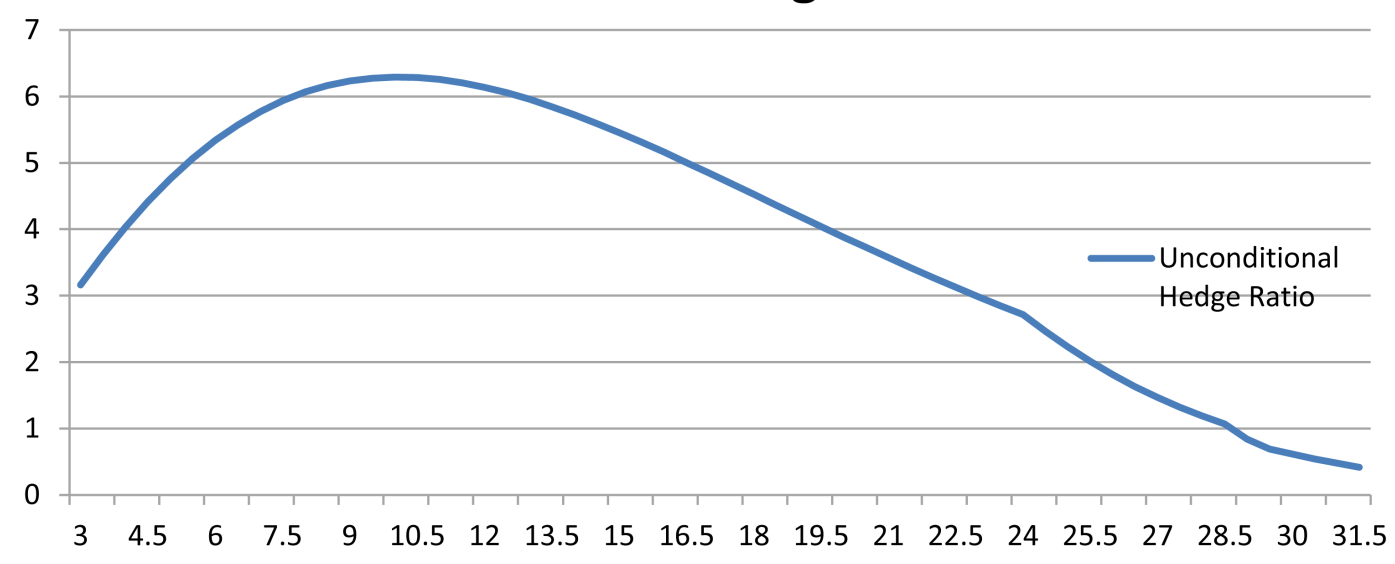

Figure 3. Unconditional hedge ratio, $k$ from 3 to 31.5 . 
(ii) Since $x_{0}=10$, we have, if $K<10$, the option is said to be in-the-money; if $K=10$, the option is atthe-money and if $K>10$, then the option is out-of-the money.

(iii) Subject to the condition (22), it can be verified that the call option price function increases (i) as time to maturity $s$ increases and (ii) as $\rho$ increases.

Delta-Neutral Portfolio

Consider the following portfolio that includes a short position of one European call with a long position delta units of the stock.

(i) The portfolio of delta-neutral positions is defined as

$$
P=-c^{*}+\Delta x_{0} \Rightarrow \text { Hedge ratio }(P)=0 .
$$

(ii) The hedge ratio expressions are similarly derived for the case of $\rho>0$ using results in Proposition 4.

\section{Conditional Put-Call Parity}

Consider a non-dividend paying European put option with strike price $K$ and exercise date s. Then the price $P\left(x_{0}, K, \rho, \eta^{2}, s\right)$ at time 0 of the put option is the present value of the expected terminal value, $E \mathrm{e}^{-r s}[K-X(s)]^{+}$, where the expectation is obtained using the risk neutral distribution of $X(s)$. Here the investor can exercise the option at time $s$ if $\ln K \geq \ln X(s)$. However we have the relationship in terms of conditional distribution of $\ln X(s)$ given $\varepsilon(s)=h$ :

$$
\begin{aligned}
& C^{*}\left(x_{0}, K, \rho, \sigma^{2}, s, h\right)-P^{*}\left(x_{0}, K, \rho, \sigma^{2}, s, h\right) \\
& =E_{0}\left(\mathrm{e}^{-r s} X(s)-\mathrm{e}^{-r s} K \mid \varepsilon(s)=h\right)=x_{0}-K \mathrm{e}^{-r s} .
\end{aligned}
$$

\section{Unconditional Call Option Price}

$$
C\left(x_{0}, K, r, \eta^{2}, s\right)=E_{0}\left(C^{*}\left(x_{0}, K, r, \eta^{2}, s, \sigma_{\varepsilon} u\right) \mid \varepsilon(s)=\sigma_{\varepsilon} u\right)=\int_{-\infty}^{\infty} C^{*}\left(x_{0}, K, r, \sigma^{2}, s, \sigma_{\varepsilon} u\right) \mathrm{e}^{-\frac{u^{2}}{2}} \mathrm{~d} u,
$$

where $\sigma_{\varepsilon}^{2}(s)=\operatorname{Var}(\varepsilon(s))$, where we have assumed the marginal distribution of $\varepsilon(s)$ to be normal with mean 0 and variance $\sigma_{\varepsilon}^{2}(s)$.

One could evaluate the option price (26) numerically as follows:

$$
\begin{aligned}
& \hat{C}_{m}\left(x_{0}, K, r, \sigma, s\right)=\sum_{j=-m}^{m} \pi_{j} C^{*}\left(x_{0}, K, r, \sigma, s, j \frac{3 \sigma_{\varepsilon}(s)}{2 m+1}\right) \\
& \sum_{j=-m}^{m} \pi_{j}=1 \\
& \pi_{j}=\Phi\left(\left(j+\frac{1}{2}\right) \frac{3 \sigma_{H}}{2 m+1}\right)-\Phi\left(\left(j-\frac{1}{2}\right) \frac{3 \sigma_{H}}{2 m+1}\right), j=0, \pm 1, \cdots,(m-1) \\
& \pi_{m}=1-\Phi\left(\left(m-\frac{1}{2}\right) \frac{3 \sigma_{H}}{2 m+1}\right) \\
& \pi_{-m}=\Phi\left(\left(m+\frac{1}{2}\right) \frac{3 \sigma_{H}}{2 m+1}\right)
\end{aligned}
$$

\section{Put-Call Parity}

The Put option price is obtained using Put-Call parity:

$$
\begin{aligned}
& P\left(x_{0}, K, \rho, \eta^{2}, s\right) \\
& =E_{H} C^{*}\left(x_{0}, K, \rho, \eta^{2}, s, h\right)-E_{H}\left[E_{0}\left(\mathrm{e}^{-r s} X(s)\right)-\mathrm{e}^{-r s} K \mid \varepsilon(s)=h\right] \\
& =E_{H} C^{*}\left(x_{0}, K, \rho, \eta^{2}, s, h\right)-\left(x_{0}-\mathrm{e}^{-r s} K\right)
\end{aligned}
$$


Again, we can apply the discrete approximation numerical method as in (26) in evaluating (27).

Figures 4-6 represent respectively, conditional call option price given $h=-0.5146,0,0.5146$.

Call option price functional values for the Equation (26) for $m=1$, as the time to maturity $s=0.5$ and the strike price Kvaries.

For $m=1,(26)$ reduces to (28):

$$
\begin{gathered}
C\left(x_{0}, K, r, \eta, \sigma_{\varepsilon}\right)=\pi_{0} C^{*}\left(x_{0}, K, r, \eta, 0\right)+\pi_{1} C^{*}\left(x_{0}, K, r, \eta, \sigma_{\varepsilon}\right)+\pi_{-1} C^{*}\left(x_{0}, K, r, \eta,-\sigma_{\varepsilon}\right), \\
\pi_{0}=0.6827, \pi_{1}=0.15865 \text { and } \pi_{-1}=0.15865 .
\end{gathered}
$$

call price- $h=-0.5146$

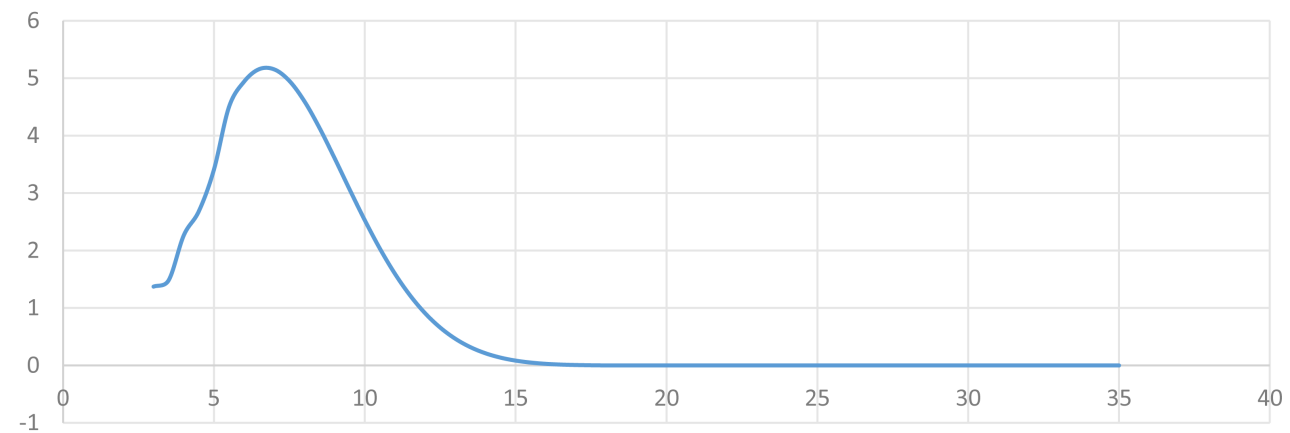

Figure 4. Conditional call price where $h=-0.5146$.

call price- $\mathrm{h}=0$

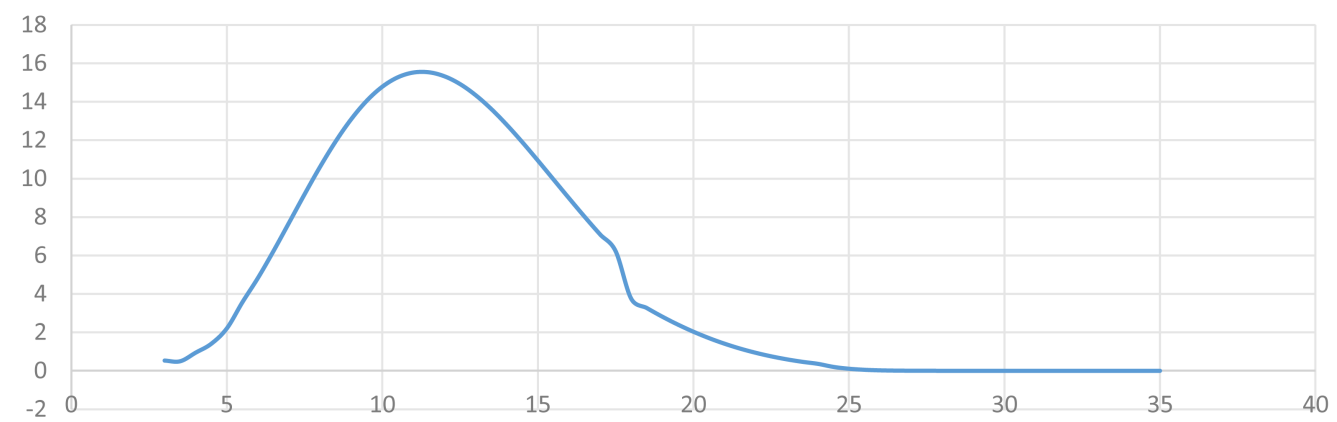

Figure 5. Conditional call price where $h=0$.

$$
\text { call price- } h=0.5146
$$

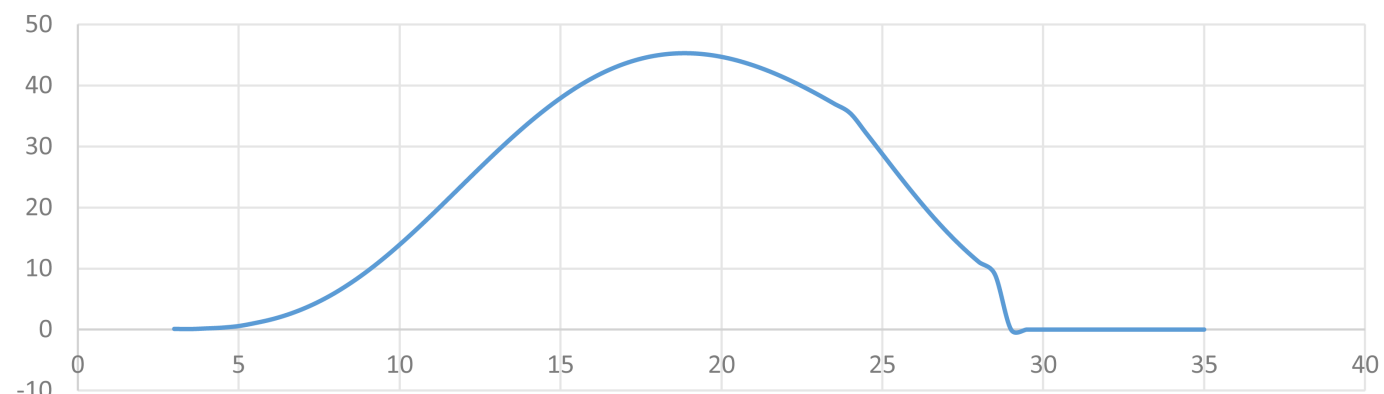

Figure 6. Conditional call price where $h=0.5146$. 
The unconditional cost of call option as a weighted average of the cost of call option, as approximated for $m=$ 1, can be represented by Figure 7.

\section{Implied Volatility Functions}

By definition, an implied volatility function is the function $\sigma^{* *}(x(0), K, r, s)$ such that the following equation, connecting the call option price $C^{*}\left(x_{0}, K, \eta^{2}, r, s\right)$ of the new model with the corresponding BlackSholes model's call option price $C_{B S}\left(x_{0}, K, \sigma^{* 2}, r, s\right)$, is satisfied, where

$$
\begin{aligned}
C\left(x_{0}, K, \eta^{2}, r, s\right)= & C_{B S}\left(x_{0}, K, \sigma^{* 2}, r, s\right)=x_{0} \Phi\left(l_{1}\left(\sigma^{*}\right)\right)-K \Phi\left(l_{2}\left(\sigma^{*}\right)\right) \\
& l_{1}(\sigma)=\frac{\ln \left(x_{0} / K\right)+r s}{\sqrt{\sigma^{2} s}}+\frac{\sqrt{\sigma^{2} s}}{2} \\
& l_{2}(\sigma)=l_{1}(\sigma)-\sqrt{\sigma^{2} s}
\end{aligned}
$$

In other words, we find a suitable value for implied volatility $\sigma^{*}$ so that call option price values both under the new model with parameter values $\left(r, \eta^{2}, x_{0}, K, s\right)$ and under the Black-Sholes model with parameters $\left(r, \sigma^{* 2}, x_{0}, K, s\right)$ are equal. Implied volatility is a popular estimate of future stock price volatility, obtained from option price data. It is known that under a Black-Scholes model formulation the implied volatility function must remain constant for different values of the strike price when the other parameters of the option pricing model are kept constant. However, skewness in implied volatility curves is observed in actual market data for European options.

With a view to explaining this anomaly, several different models have been proposed in the option-price literature. These models are mostly variations of 2-factor affine-jump diffusion models, one of the factors being stock volatility ${ }^{3}$

$$
\text { Let }\left(x_{0}, r, \alpha, \eta, \kappa, \theta, v, \rho\right)=(10,0.06,1,0.6,0.2,0.05,0.08,-0.8)
$$

In this section, we show that the implied volatility skewness property of negative correlation- $\rho<0$ model. The "implied volatility smile curves are rotated clock wise into smirks", which is known as "Volatility asymmetry". See [4], p. 350. The implied volatility can be easily computed and is an increasing function of the time to maturity $s$-(see Figure 8).

\section{Conclusion}

In this paper, we formulate a two-factor model of a stock index, where we assume the volatility process and the Brownian motion process of the model are dependent and use a novel linear regression approach to obtain call option price expressions for the proposed model. We have obtained closed form Black-Scholes type expressions

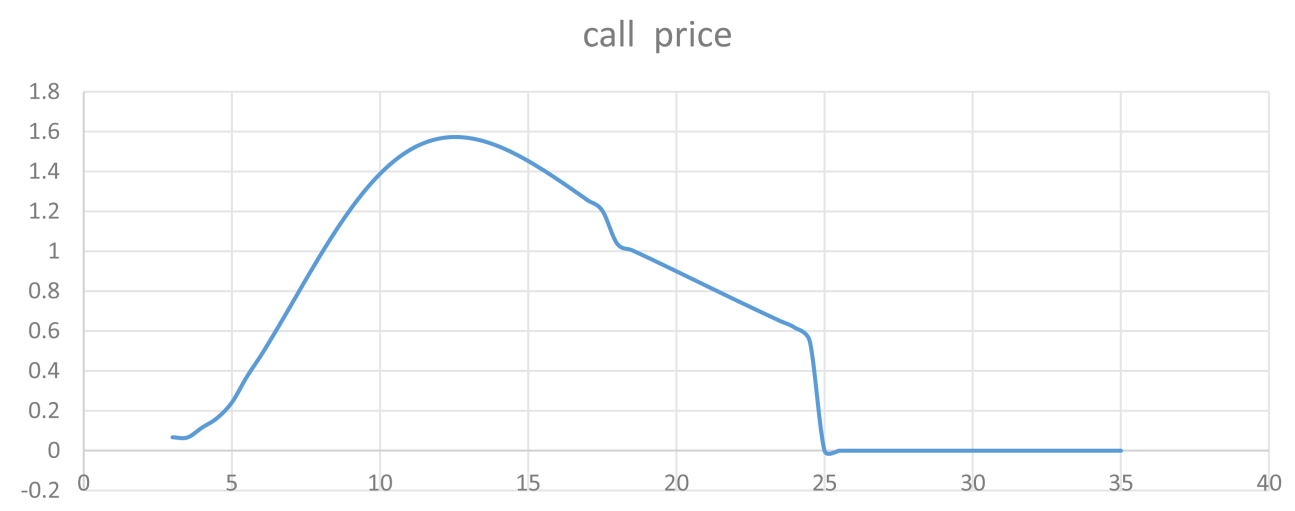

Figure 7. Unconditional call option, $k$ from 3 to 35 .

\footnotetext{
$\overline{{ }^{3} \text { But there are several empirical papers that use } S \& P} 500$ options data-set on a given date directly to estimate risk-neutral return densities and a measure of risk-neutral skewness, [17]-[20].
} 


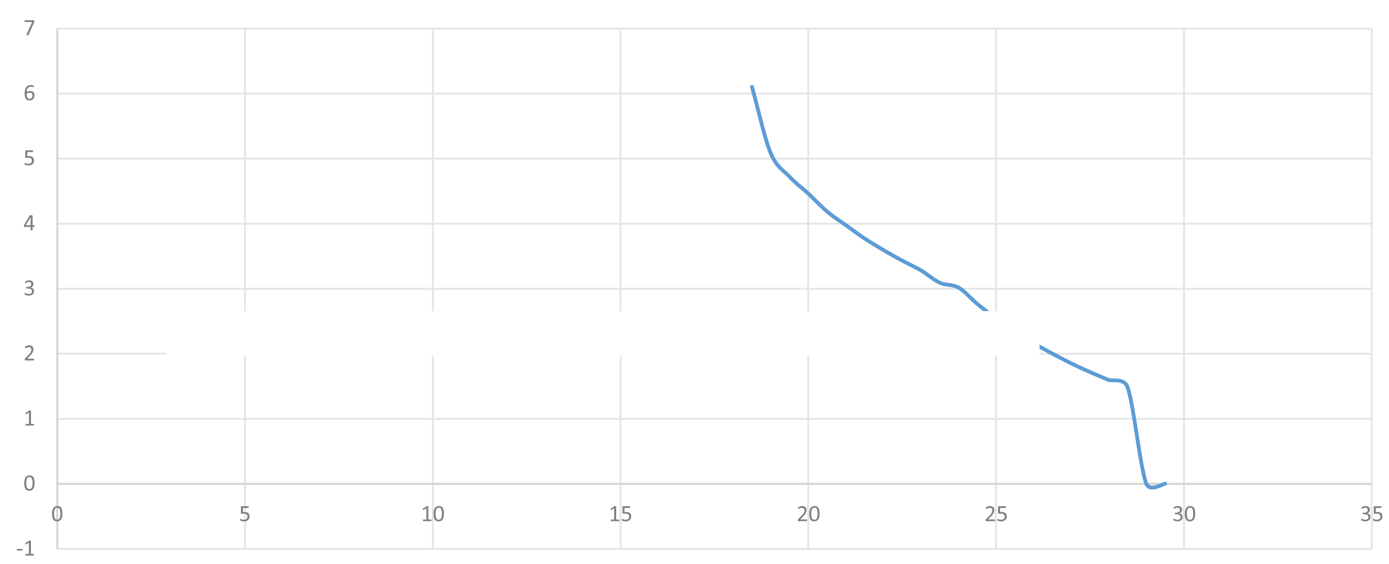

Figure 8. Implied volatility.

for option prices under the assumption of constant interest rate. We can also show stochastic interest rate and random economic shocks can also be incorporated in the model (see [21]-[23]). Analyzing the proposed model is computationally simpler than it is for the other affine jump process models. The results of this paper can also be applied to bond option, foreign currency option and futures option models and to more complex derivative securities including various types of mortgage-backed securities.

\section{References}

[1] Cox, J.C. and Ross, S.A. (1976) The Valuation of Options for Alternative Stochastic Processes. Journal of Financial Economics, 3, 145-166. http://dx.doi.org/10.1016/0304-405X(76)90023-4

[2] Heston, S. (1993) A Closed-Form Solution for Options with Stochastic Volatility with Applications to Bond and Currency Options. Review of Financial Studies, 6, 327-344. http://dx.doi.org/10.1093/rfs/6.2.327

[3] Pan, J. (2002) The Jump-Risk Premia Implicit in Options Evidence from an Integrated Time Series. Journal of Financial Economics, 63, 3-50. http://dx.doi.org/10.1016/S0304-405X(01)00088-5

[4] Duffie, D. and Singleton, K. (2003) Credit Risk: Pricing, Measurement, and Management. Princeton University Press, Princeton, 350.

[5] Hull, J.C. and White, A. (1987) The Pricing of Options on Assets with Stochastic Volatilities. The Journal of Finance, 42, 281-300. http://dx.doi.org/10.1111/j.1540-6261.1987.tb02568.x

[6] Jarrow, R., Li, H. and Zhao, F. (2007) Interest Rate Caps "Smile" Too! But Can the "LIBOR" Market Models Capture the Smile? The Journal of Finance, 62, 345-382. http://dx.doi.org/10.1111/j.1540-6261.2007.01209.x

[7] Duffie, D. (1996) Dynamic Asset Pricing Theory. 2nd Edition, Princeton University Press, Princeton.

[8] Cox, J.C., Ross, S.A. and Rubinstein, M. (1979) Option Pricing: A Simplified Approach. Journal of Financial Economics, 7, 229-263.

[9] Figlewski, S. and Gao, B. (1999) The Adaptive Mesh Model, a New Approach to Efficient Option Pricing. Journal of Financial Economics, 53, 313-351. http://dx.doi.org/10.1016/S0304-405X(99)00024-0

[10] Cumby, R., Figlewski, S. and Hashbrook, J. (1993) Forecasting Volatilities and Correlations with EGARCH Models. The Journal of Derivatives, 1, 51-63. http://dx.doi.org/10.3905/jod.1993.407877

[11] Campbell, J.Y., Lo, A.W. and MacKinlay, A.C. (1997) The Econometrics of Financial Markets. Princeton University Press, Princeton.

[12] Øksendal, B. (2003) Stochastic Differential Equations: An Introduction with Applications. Springer-Verlag, Berlin.

[13] Nelson, D.B. (1991) Conditional Heteroskedasticity in Asset Returns: A New Approach. Econometrica, 50, $347-370$. http://dx.doi.org/10.2307/2938260

[14] Black, F., Derman, E. and Toy, W. (1990) A One-Factor Model of Interest Rates and Its Application to Treasury-Bond Options. Financial Analysts Journal, 46, 33-39. http://dx.doi.org/10.2469/faj.v46.n1.33

[15] Trolle, A.B. and Schwarz, E.S. (2009) A General Stochastic Volatility Model for the Pricing of the Interest Rate Derivatives. The Review of Financial Studies, 22, 2007-2057.

[16] Vasicek, O. (1977) An Equilibrium Characterization of the Term Structure. Journal of Financial Economics, 5, 177188. 
[17] Ait-Sahalia, Y. and Lo, A. (1998) Non-Parametric Estimation of State-Price Densities Implicit in Financial Asset Prices. The Journal of Finance, 53, 499-547. http://dx.doi.org/10.1111/0022-1082.215228

[18] Bakshi, G., Cao, C. and Chen, Z. (1997) Empirical Performance of Alternative Option Pricing Models. The Journal of Finance, 52, 2003-2049. http://dx.doi.org/10.1111/j.1540-6261.1997.tb02749.x

[19] Bakshi, G., Kapadia, N. and Madan, D. (2003) Stock Return Characteristics, Skew Laws, and the Differential Pricing of Individual Equity Options. The Review of Financial Studies, 16, 101-143. http://dx.doi.org/10.1093/rfs/16.1.0101

[20] Buraschi, A. and Jackwerth, J.C. (2001) The Price of a Smile: Hedging and Spanning in Option Markets. The Review of Financial Studies, 14, 495-527. http://dx.doi.org/10.1093/rfs/14.2.495

[21] Merton, R.C. (1976) Option Pricing when the Underlying Stock Returns Are Discontinuous. Journal of Financial Economics, 3, 125-144. http://dx.doi.org/10.1016/0304-405x(76)90022-2

[22] Jagannathan, R. (2008) A Class of Asset Pricing Models Governed by Subordinated Processes that Signal Economic Shocks. Journal of Economic Dynamics and Control, 32, 3820-3846.

[23] Scott, L.O. (1997) Pricing Stock Options in a Jump-Diffusion Model with Stochastic Volatility and Interest Rates: Applications of Fourier Inversion Methods. Mathematical Finance, 7, 413-426. http://dx.doi.org/10.1111/1467-9965.00039

[24] Wilks, S.S. (1962) Mathematical Statistics. John Wiley \& Sons, Inc., Hoboken. 


\section{Appendix}

\section{Appendix A}

Some preliminary results are stated below prior to the proof of Proposition 1 .

Application of Least Squares Linear Regression (see [24], p. 87).

$$
q^{*}(s) \triangleq \int_{0}^{s} q_{0}(u) \mathrm{d} B_{1}(u) \text { on } B_{1}(s)=\int_{0}^{s} \mathrm{~d} B_{1}(u),
$$

where $q_{0}(u)=(\kappa-\theta) \mathrm{e}^{-\alpha u}$.

The regression equation obtained is:

$$
q^{*}(s)=\int_{0}^{s} q_{0}(u) \mathrm{d} B_{1}(u)=\gamma_{1}(s) B_{1}(s)+e_{1}(s)
$$

and where

$$
\gamma_{1}(s)=\frac{\operatorname{cov}\left(\int_{0}^{s} q_{0}(u) \mathrm{d} B_{1}(u), \int_{0}^{s} \mathrm{~d} B_{1}(u)\right)}{\operatorname{var}\left(\int_{0}^{s} \mathrm{~d} B_{1}(u)\right)}=\left[\frac{1}{s} \int_{0}^{s} q_{0}(u) \mathrm{d} u\right]=\frac{(\kappa-\theta)\left(1-\mathrm{e}^{-\alpha s}\right)}{\alpha s}
$$

is the regression coefficient

$$
\begin{gathered}
e_{1}(s)=\int_{0}^{s}\left(q_{0}(u)-\gamma_{1}(s)\right) \mathrm{d} B_{1}(u) \sim N\left(0,\left[\int_{0}^{s}\left(q_{0}^{2}(u) \mathrm{d} u-\gamma_{1}(s)\right)^{2}\right]^{\frac{1}{2}}\right) . \\
\operatorname{Var}\left\{e_{1}(s)\right\}=(\kappa-\theta)^{2}\left[\left(\frac{1-\mathrm{e}^{-2 \alpha s}}{2 \alpha}\right)-\left(\frac{1-\mathrm{e}^{-\alpha}}{\alpha s}\right)^{2}\right] .
\end{gathered}
$$

2) Regress the function

$$
\psi^{* *}(s) \triangleq \int_{0}^{s} \mathrm{~d} B_{1}(u) \int_{0}^{u} \psi(t) \mathrm{d} B_{1}(t) \text { on } \int_{0}^{s} \mathrm{~d} B_{1}(u) \int_{0}^{u} \mathrm{~d} B_{1}(t)
$$

Note that (see [12])

$$
\int_{0}^{s} \mathrm{~d} B_{1}(u) \int_{0}^{u} \mathrm{~d} B_{1}(t)=\int_{0}^{u} B_{1}(u) \mathrm{d} B_{1}(u)
$$

We can show that (see [12])

$$
\int_{0}^{u} B_{1}(u) \mathrm{d} B_{1}(u)=\left(B^{2}(s)-s\right) / 2 .
$$

\section{Proof:}

Using Ito's Lemma, we have

$$
\begin{aligned}
& \mathrm{d} B^{2}=2 B \mathrm{~d} B+\mathrm{d} u \\
& \therefore \int_{0}^{s} \mathrm{~d} B^{2}=2 \int_{0}^{s} B \mathrm{~d} B+\int_{0}^{s} \mathrm{~d} u .
\end{aligned}
$$

This completes the proof.

$$
\begin{aligned}
& \gamma_{2}(s)=\frac{2}{s^{2}} \int_{0}^{s} \mathrm{~d} u \int_{0}^{u} \psi(t) \mathrm{d} t=\frac{2}{s^{2}} \eta \int_{0}^{s} \frac{1-\mathrm{e}^{-\alpha u}}{\alpha} \mathrm{d} u=\frac{2}{s^{2}} \eta \frac{\left(s-\left(1-\mathrm{e}^{\alpha s}\right) / \alpha\right)}{\alpha}=\frac{2}{s^{2}} \eta \frac{\left(\alpha s-\left(1-\mathrm{e}^{\alpha s}\right)\right)}{\alpha^{2}} . \\
& \gamma_{2}(s)=\left[\frac{2}{s^{2}} \int_{0}^{s} \mathrm{~d} u \int_{0}^{u} \psi(t) \mathrm{d} t\right]
\end{aligned}
$$


is the regression coefficient

$$
\begin{gathered}
e_{2}(s)=\frac{2}{s^{2}} \int_{0}^{s} \int_{0}^{u}\left(\psi(t)-\gamma_{2}(s)\right) \mathrm{d} B_{1}(t) \mathrm{d} B_{1}(u) . \\
E\left(e_{2}(s)\right)=0 ; E\left(e_{1}(s)\right)=0, \operatorname{Cov}\left(e_{1}(s), e_{2}(s)\right)=0 \\
\operatorname{Var}\left(e_{2}(s)=\left[\int_{0}^{s} \mathrm{~d} u \int_{0}^{u}\left(\psi(t)-\gamma_{2}(s)\right)^{2} \mathrm{~d} t\right]\right)=\frac{2}{s^{2}} \int_{0}^{s} \int_{0}^{u} \psi^{2}(t) \mathrm{d} t \mathrm{~d} u-\gamma_{2}^{2}(s)
\end{gathered}
$$

Then the regression equation is

$$
\psi^{* *}(s)=\gamma_{2}(s)\left(B_{1}^{2}(s) / 2-\frac{s}{2}\right)+e_{2}(s)
$$

Assumption:

$$
e_{2}(s) \approx N\left(0, \operatorname{Var}^{1 / 2}\left(e_{2}(s)\right)\right) \text { (approximately) }
$$

Note that $\operatorname{Cov}\left(e_{1}(s), e_{2}(s)\right)=0$ and $e_{1}(s) \approx N\left(0, \operatorname{Var}^{1 / 2}\left(e_{2}(s)\right)\right)$.

$$
\therefore\left(\operatorname{Var}\left(e_{1}(s)\right)+\operatorname{Var}\left(e_{2}(s)\right)^{1 / 2}\right)=\operatorname{Var}\left(e_{1}(s)\right)+\operatorname{Var}\left(e_{2}(s)\right)
$$

\section{Assumption:}

$$
\varepsilon(s)=e_{1}(s)+e_{2}(s) \sim N\left(0,\left(\operatorname{Var}\left(e_{1}(s)\right)+\operatorname{Var}\left(e_{2}(s)\right)\right)^{1 / 2}\right) \quad \text { (approximately) }
$$

\section{Proof of Proposition 1:}

1)

$$
\begin{aligned}
& \mathrm{d}[\ln X(u)]=\left(r-\frac{v^{2}}{2}\right) \mathrm{d} u+\int_{0}^{u}\left[v+q(u)+\psi(t)\left(\rho \mathrm{d} B_{1}(t)+\delta \mathrm{d} C_{1}(t)\right)\right] \mathrm{d} B_{1}(u), 0 \leq u \leq s \\
& \int_{0}^{s} \mathrm{~d}[\ln X(s)]=\int_{0}^{s} \mathrm{~d} u\left(r-\frac{v^{2}}{2}\right)+\int_{0}^{u}\left(v+\theta+q_{0}(u)+\psi(t)\left(\rho \mathrm{d} B_{1}(t)+\delta \mathrm{d} C_{1}(t)\right)\right) \mathrm{d} B_{1}(u) \\
& \ln X_{s}=\ln x_{0}+r s-\frac{1}{2} v^{2} s+\left[(v+\theta) B_{1}(s)+\left[\gamma_{1}(s) B_{1}(s)+e_{1}(s)\right]\right. \\
&\left.+\left[\gamma_{2}(s) \rho\left(\frac{B_{1}^{2}(s)}{2}-\frac{s}{2}\right)+e_{2}(s)\right]+\delta \bar{V} B_{1}(s)\right] \\
&=\ln x_{0}+r s-\frac{1}{2} v^{2} s+(v+\theta) \rho\left(\frac{B_{1}^{2}(s)}{2}-\frac{s}{2}\right)+\left[\gamma_{1}(s) B_{1}(s)+e_{1}(s)\right] \\
&+\delta \bar{V} B_{1}(s)+\rho\left[\gamma_{2}(s)\left(\frac{B_{1}^{2}(s)}{2}-\frac{s}{2}\right)+e_{2}(s)\right]
\end{aligned}
$$

2)

$$
\int_{0}^{s} \mathrm{~d} B_{1}(u) \int_{0}^{u} \psi(t) \mathrm{d} B_{1}(t)=\gamma_{2}(s) \int_{0}^{s} B_{1}(u) \mathrm{d} B_{1}(u)+e_{2}(s)=\gamma_{2}(s)\left(B_{1}^{2}(s)-\frac{s}{2}\right)+e_{2}(s)
$$

where

$$
\gamma_{2}(s)=\frac{2}{s^{2}} \int_{0}^{s} \mathrm{~d} u \int_{0}^{u} \psi(t) \mathrm{d} t=\frac{2}{s^{2}} \eta \int_{0}^{s} \frac{1-\mathrm{e}^{-\alpha u}}{\alpha} \mathrm{d} u=\frac{2}{s^{2}} \eta \frac{\left(s-\left(1-\mathrm{e}^{\alpha s}\right) / \alpha\right)}{\alpha}=\frac{2}{s^{2}} \eta \frac{\left(\alpha s-\left(1-\mathrm{e}^{\alpha s}\right)\right)}{\alpha^{2}}
$$




$$
e_{2}(s)=\int_{0}^{s}\left(\psi^{*}(u)-\gamma_{2}(s)\right) \mathrm{d} u
$$

where

$$
\begin{aligned}
& \psi^{*}(u) \triangleq \int_{0}^{u} \psi(t) \mathrm{d} t . \\
& \operatorname{Var}\left(e_{2}(s)\right)=\int_{0}^{s}\left(\psi^{*}(u)\right)^{2} \mathrm{~d} t \mathrm{~d} u-\gamma_{2}^{2}(s)=\int_{0}^{s}\left(\eta^{2} \frac{1-\mathrm{e}^{-2 \alpha u}}{2 \alpha}\right) \mathrm{d} u-\left[\frac{2}{s} \eta \frac{\left(s-\left(1-\mathrm{e}^{\alpha s}\right) / \alpha\right)}{\alpha}\right]^{2}
\end{aligned}
$$

\section{Appendix B}

\section{Proof of Proposition 3:}

$$
P(\ln X(s) \geq \omega \mid \varepsilon(s)=h)=P\left(m(s)\left(Z-z_{1}\right)\left(Z-z_{2}\right) \geq 0\right)
$$

Now we assume $m(s)>0$ and $z_{1} \geq z_{2}$. Then

$$
\begin{aligned}
& P(\ln X(s) \geq v \mid H(s)=h)=P\left(\left(Z \geq z_{1}\right) \cup\left(Z \leq z_{2}\right)\right)=1-\Phi\left(z_{1}\right)+\Phi\left(z_{2}\right) \\
& P(\ln X(s) \leq v \mid H(s)=h)=\Phi\left(z_{1}\right)-\Phi\left(z_{2}\right)
\end{aligned}
$$

If $n^{2}(s)-2 m(s) p(s, h)=0$, then the roots of the equation defined in (18) are equal so that $z_{1}=z_{2}=z^{*}=-\frac{n(s)}{m(s)}$, then there exists a value $\omega^{*}(\rho, h)$ such that

$$
P\left(\ln X(s) \geq \omega^{*}(\rho, h) \mid \varepsilon(s)=h, C(s)=\xi\right)=1
$$

In other words, $\omega^{*}(\rho, h)$ is the lowest value the conditional random variable $\{\ln X(s) \mid \varepsilon(s)=h\}$ can assume.

The equations defined in (12) hold under the Assumption (2) so that the roots of the quadratic Equation (13) are well defined.

Substituting for $p(s, h)$ in the condition:

$$
\begin{gathered}
n^{2}(s)-2 m(s) p(s, h) \geq 0, \text { we have } \\
n^{2}(s)-2 m(s) p(s, h)=n^{2}(s)-2 m(s)\left(p^{*}(s, h)+\ln \left(x_{0}\right)-r s\right) \geq-2 m(s) \omega, \text { where } \\
p(s, h)=p^{*}(s, h)+\ln \left(x_{0}\right)-r s \\
\rho>0 \Leftrightarrow m(s)>0 \Rightarrow \omega^{*}(\rho, h)=\left[p^{*}(s, h)+\ln \left(x_{0}\right)-r s-\frac{n^{2}(s)}{2 m(s)}\right] \leq \omega . \\
\rho<0 \Leftrightarrow m(s)<0 \Rightarrow \omega^{*}(\rho, h)=\left[p^{*}(s, h)+\ln \left(x_{0}\right)-r s-\frac{n^{2}(s)}{2 m(s)}\right] \geq \omega .
\end{gathered}
$$

In other words $\omega^{*}(\rho, h)$ is the highest value the conditional random variable $\{\ln X(s) \mid \varepsilon(s)=h\}$ can assume.

An explicit expression for $\omega^{*}(\rho, h)$ 


$$
\omega^{*}(\rho, h)=p^{*}(s, h)+\ln \left(x_{0}\right)-r s-\frac{n^{2}(s)}{2 m(s)}
$$

Then, $P\left(\ln X(s) \geq \omega^{*}(\rho, h)\right)=1$.

$$
p^{*}(s, h)=p(s, h)-r s-\ln x_{0}+\ln K
$$

